\title{
Muito Além de uma Coleção de Plantas: Contribuições do Herbário Professor Aluízio Bittencourt (HABIT), para o Conhecimento da Flora do Maranhão, Brasil
}

\author{
Far Beyond of a Collection of Plants: Contributions of Professor Aluízio \\ Bittencourt Herbarium (HABIT) to the Knowledge of Flora Maranhão, Brazil
}

\author{
Alex Medeiros Silva', Domingos Lucas dos Santos-Silva², Guilherme Sousa da Silva ${ }^{3}$, Gustavo \\ da Silva Gomes ${ }^{4}$, Regigláucia Rodrigues Oliveira ${ }^{5}$, Ronison Ferreira Oliveira ${ }^{6}$, Gonçalo Mendes \\ da Conceição ${ }^{7}$
}

'Laboratório de Biologia Vegetal. Universidade Estadual do Maranhão, Centro de Estudos Superiores de Caxias, Praça Duque de Caxias, Morro do Alecrim, s/n, 65.604-380, Caxias, MA, Brasil. http://orcid.org/0000-0002-3690-8702, E-mail: studios_lex@hotmail.com

${ }^{2}$ Laboratório de Ecologia. Universidade do Estado de Mato Grosso, Avenida Perimetral Rogério Silva, 4930, Jardim Flamboyant, 78580-000, Alta Floresta, MT, Brasil. http://orcid.org/0000-0001-6700-6659, E-mail:domingoslukas@gmail.com

3 Instituto de Biologia. Universidade Estadual de Campinas, Rua Monteiro Lobato, 255, 13083-862, Campinas, SP, Brasil. http://orcid.org/00000002-4250-0017, E-mail: guilhermecx.cx@hotmail.com

${ }^{4}$ Laboratório de Biologia Vegetal. Universidade Estadual do Maranhão, Centro de Estudos Superiores de Caxias, Praça Duque de Caxias, Morro do Alecrim, s/n, 65.604-380, Caxias, MA, Brasil. http://orcid.org/0000-0002-1640-5462, E-mail: gustavocjk@gmail.com

${ }^{5}$ Universidade Estadual do Maranhão, Centro de Estudos Superiores de Zé Doca, Rua Rio Branco, Centro, s/n, 65.365-000, Zé Doca, MA, Brasil. http://orcid.org/0000-0003-3916-3686, E-mail: regiglaucia@hotmail.com

${ }^{6}$ Laboratório de Biologia Vegetal. Universidade Estadual do Maranhão, Centro de Estudos Superiores de Caxias, Praça Duque de Caxias, Morro do Alecrim, s/n, 65.604-380, Caxias, MA, Brasil. http://orcid.org/0000-0001-8393-2126, E-mail: ronybiogtr@gmail.com

${ }^{7}$ Laboratório de Biologia Vegetal. Universidade Estadual do Maranhão, Centro de Estudos Superiores de Caxias, Praça Duque de Caxias, Morro do Alecrim, s/n, 65.604-380, Caxias, MA, Brasil. http://orcid.org/0000-0001-9056-9070,E-mail: doutorgoncalo@gmail.com *Autor para correspondência

\section{Palavras-chave \\ Coleção Biológica \\ Herbários do Maranhão \\ Popularização da Botânica}

O artigo documenta e descreve de forma geral as contribuições do Herbário Professor Aluízio Bittencourt (HABIT), no mapeamento da biodiversidade e na geração de conhecimento florístico do Maranhão para profissionais, estudantes e comunidade em geral, e ainda encoraja futuros pesquisadores das diferentes áreas da ciência a inserção das coleções botânicas, como modelo de estudo. A coleção e as atividades realizadas pelo HABIT contribuem de forma direta e indireta para o conhecimento e conservação da biodiversidade local e regional do Maranhão. Assim, é necessário a realização de trabalhos que se baseiam em listas florísticas de coleções botânicas, aperfeiçoamento de profissionais para atuarem em manutenção de coleções, frente aos avanços tecnológicos e científicos. Consequentemente, é indispensável a aplicação de recursos financeiros em coleções botânicas para a catalogação e continuidades das atividades que estruturam, moldam e documentam o conhecimento da biodiversidade global.

\section{Keywords}

Biological Collection Maranhão herbaria Popularization of botany
This article documents and describes in general the contributions of the Professor Aluízio Bittencourt Herbarium (HABIT) in the mapping of biodiversity and in the generation of floristic knowledge of Maranhão for professionals, students and the community in general, still encourages future researchers from different spheres of science to insert of the botanical collections as a model of study. The collection and activities carried out by HABIT contribute directly and indirectly to the knowledge and conservation of the local and regional biodiversity of Maranhão. Thus, it is necessary to carry out works that are based on floristic lists of botanical collections, improvement of professionals to work in the maintenance of collections in the face of technological and scientific advances. Consequently, it is indispensable to apply financial resources in botanical collections for the cataloguing and continuity of the activities that structure, shape and document the knowledge of global biodiversity.

\section{INTRODUÇÃO}

A biodiversidade do Maranhão, encontra-se cada vez mais ameaçada, devido a degradação florestal, queimadas, conversão do uso da terra e fatores socioeconômicos (CELENTANO, 2018). Dessa forma, há uma necessidade crescente de se conhecer a biodiversidade ainda não identificada e conservar a registrada nos variados 
Alex Medeiros Silva, Domingos Lucas dos Santos-Silva, Guilherme Sousa da Silva, Gustavo da Silva Gomes, Regigláucia Rodrigues Oliveira, Ronison Ferreira Oliveira, Gonçalo Mendes da Conceição

ecossistemas maranhenses, que vão desde ambientes salinos (manguezais), babaçuais até ambientes vegetacionais, com característica dos domínios fitogeográficos do Cerrado, Caatinga e Amazônia (MUNIZ, 2006; ALVES et al. 2009; OLíMPIO et al. 2016; SANTOS-SILVA et al. 2018). Assim, a preservação de espécimes é um importante meio para garantir o conhecimento da biodiversidade para as próximas gerações (SEGOVIA-SALCEDO et al. 2015).

Estima-se que 6.500 coleções de museus e instituições acadêmicas possuem mais de 3 bilhões de espécimes preservando a história natural da Terra (SIMMONS; MUÑOZSABA, 2005). Entretanto, as coleções biológicas não constituem apenas um acervo de amostras de vouchers, mas também é um recurso essencial para a educação e formação científica de profissionais da área de Ciências Biológicas (COOK et al. 2014). Além disso, podem ser um dos meios mais eficazes para traçar metas de conservação da biodiversidade (CASTILLO-FIGUEROA, 2018), como por exemplo as coleções botânicas, os "herbários" (GREVE et al. 2016; BESNARD et al. 2018).

Para o estado do Maranhão, esses acervos científicos de plantas secas se tornam indispensáveis para compreender e estudar a distribuição da biodiversidade vegetal presente nos diferentes tipos de ecossistemas do estado (MARTINS et al. 2017; NASCIMENTO et al. 2018; SILVA et al. 2019), já que apresentam coleções de espécimes vegetais amostrados em diferentes anos, locais e estarem associados com os dados de coletas (JOYE et al. 2002; LE BRAS et al. 2017).

São registrados no Maranhão, cinco herbários, com aproximadamente 30.000 espécimes vegetais (Figura 1; NYBG, 2020; SBB, 2020). Esses herbários apresentam importância muito além das quais foram destinados, pois além de resguardar o conhecimento da flora maranhense, contribuem para a formação de recursos humanos e científicos (ALMEIDA JR., 2015; CONCEIÇÃO, 2015).

Neste estudo, documenta-se o histórico e destaca-se as contribuições do Herbário Prof. ${ }^{\circ}$ Aluízio Bittencourt (HABIT), no mapeamento da biodiversidade e na geração de conhecimento florístico do Maranhão para profissionais, estudantes e comunidade em geral e ainda encoraja futuros pesquisadores das diferentes áreas da ciência a inserirem as coleções botânicas como modelo de estudo.

\section{SOBRE O HERBÁRIO PROFESSOR ALUÍZIO BITTENCOURT/HABIT}

\section{Histórico e Infraestrutura}

A iniciativa para criação de uma coleção botânica, no Centro de Estudos Superiores de Caxias/CESC, da Universidade Estadual do Maranhão/UEMA, se iniciou em meados de 1970 com atividades de docência do Professor Deusiano Bandeira de Almeida, quando o CESC/UEMA, ainda era a Faculdade de Formação de Professores do Ensino Médio (FFPEM). Mediante as aulas-práticas e teóricas de botânica, fazia-se necessário a implementação de uma coleção de plantas como acervo didático, promovida pelas atividades botânicas realizadas, por meio de coleta, herborização, montagem de exsicatas e identificação de espécies.

Em agosto de 1991, diante do acervo de amostras botânicas didáticas sob a coordenação do Prof. Deusiano Bandeira de Almeida, foi criado uma coleção botânica didático chamado de Prof. Aylton Brandão Joly, nas dependências do Laboratório de Biologia, do Centro de

Figura 1. Herbários do Maranhão registrados na Rede Brasileira de Herbários, gerenciados pela Sociedade Brasileira de Botânica.

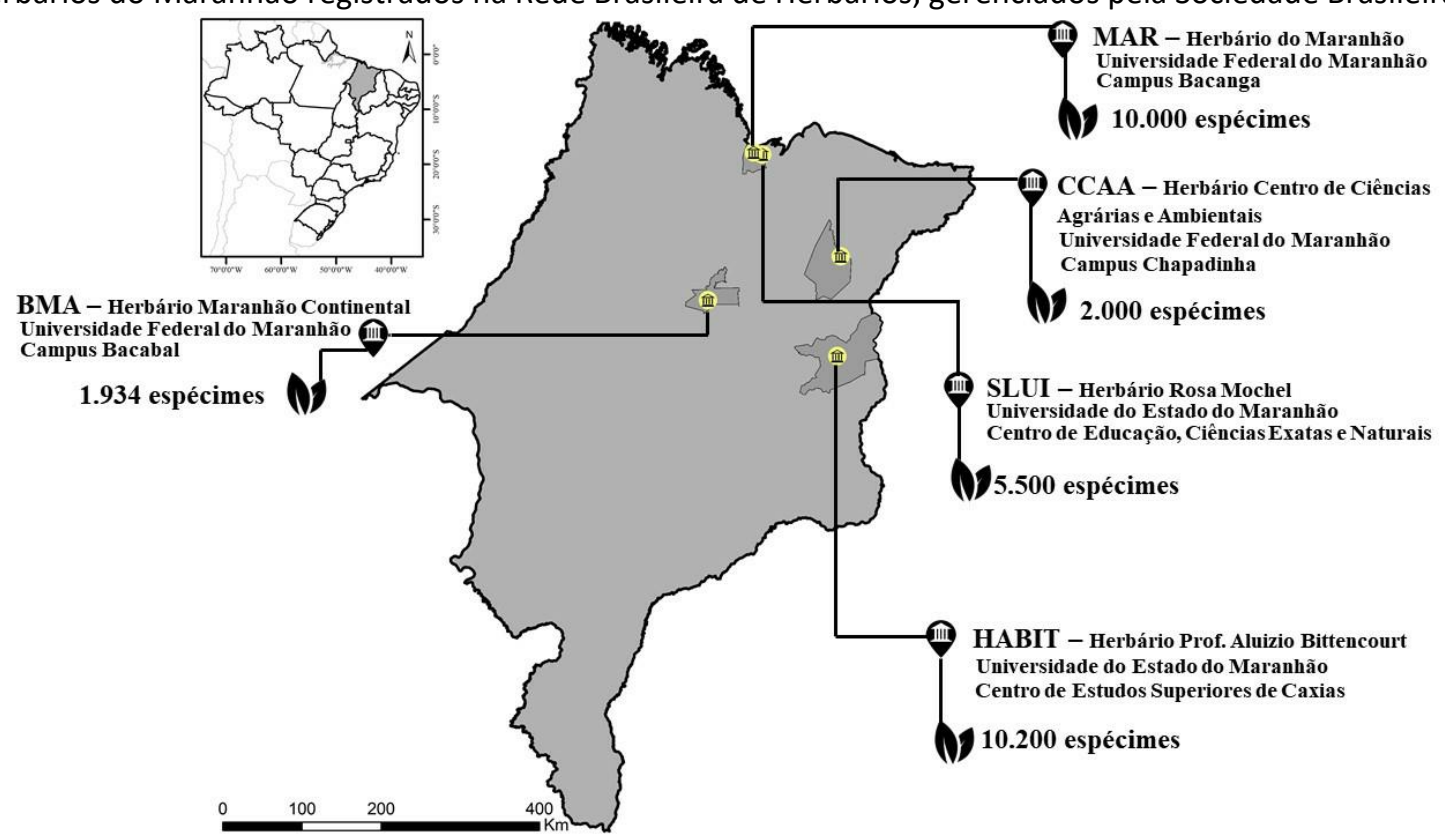


Estudos Superiores de Caxias, antiga Unidade de Estudos de Educação de Caxias. Após reformas administrativas da Universidade Estadual do Maranhão, no ano de 1994, onde a Unidade de Estudos de Educação, passou a ser denominado, de Centro de Estudos Superiores de Caxias, passando a formar mais alunos nos cursos de Ciências, onde muitos desses contribuíram, alimentando com informações sobre a flora do Maranhão, em especial do município de Caxias/MA.

Em 2006, os professores Deusiano Bandeira de Almeida e Gonçalo Mendes da Conceição, com a necessidade de um espaço especializado para o tratamento do acervo botânico, transferiu a coleção botânica Prof. Aylton Brandão Joly, do Laboratório de Biologia, para o Laboratório de Biologia Vegetal/LABIVE. A coleção, então passou a ficar resguardada no Herbário nomeado Prof. Aluízio Bittencourt/HABIT, com o intuito de homenagear o presidente da XXI Reunião Nordestina de Botânica, realizada no município de Caxias/MA, em 1997; ex-diretor do CESC/UEMA e professor que contribuiu com a criação dos cursos de História, Geografia, Pedagogia, Letras, Medicina, Enfermagem, Ciências nas habilitações em Química, Biologia, Física e Matemática e implantação de laboratórios didáticos e de pesquisas no CESC/UEMA.

O constante desenvolvimento de atividades em campo, elevou consideravelmente a quantidade de espécimes vegetais armazenados no HABIT, além de acervar outras pequenas coleções, como carpoteca e laminário, fez como que resultasse na demanda por um espaço ampliado e exclusivo para melhor abrigar e conservar as amostras. Assim, em 2018 houve a ampliação do espaço físico, no qual a coleção passou a contar com melhores estruturas para continuação do enriquecimento sobre o conhecimento da flora maranhense, aumentando também a quantidade de amostras depositadas oriundas da região, bem como aquelas doadas por outras instituições.

O novo local do HABIT (Figura 2) foi estruturado com uma sala laboratorial para análise e identificação de material herborizado, sala de informática e cadastro de espécie, sala da curadoria, uma sala para a coleção botânica e um depósito para guardar o material de campo. Fazendo com que o objetivo de armazenar informações sobre espécies da flora do estado do Maranhão seja alcançado.

\section{A Coleção}

O HABIT possui cerca de 10.200 exsicatas, registradas no acervo do herbário, da coleção de Briófitas (ca. 3.500), Samambaias, Licófitas (ca. 100) e Angiospermas (ca. 6.500), correspondendo mais de 1.000 espécies para a Flora Maranhense. Dessas, 3.518 encontram-se no banco de dados online do Centro de Referência em Informação Ambiental (CRIA, 2020).

Dessa forma o HABIT encontra-se cadastrado na Rede Brasileira de Herbários, coordenada pela Sociedade Botânica do Brasil (SBB, 2020) e Index Herbariorum (NYBG, 2020) e se dedica em georreferenciar, armazenar, preservar, documentar e identificar a biodiversidade maranhense, com a finalidade de ensino, pesquisa e extensão.

Figura 2. Localização do Herbário Professor Aluízio Bittencourt/HABIT, do Centro de Estudos Superiores de Caxias, Universidade Estadual do Maranhão.

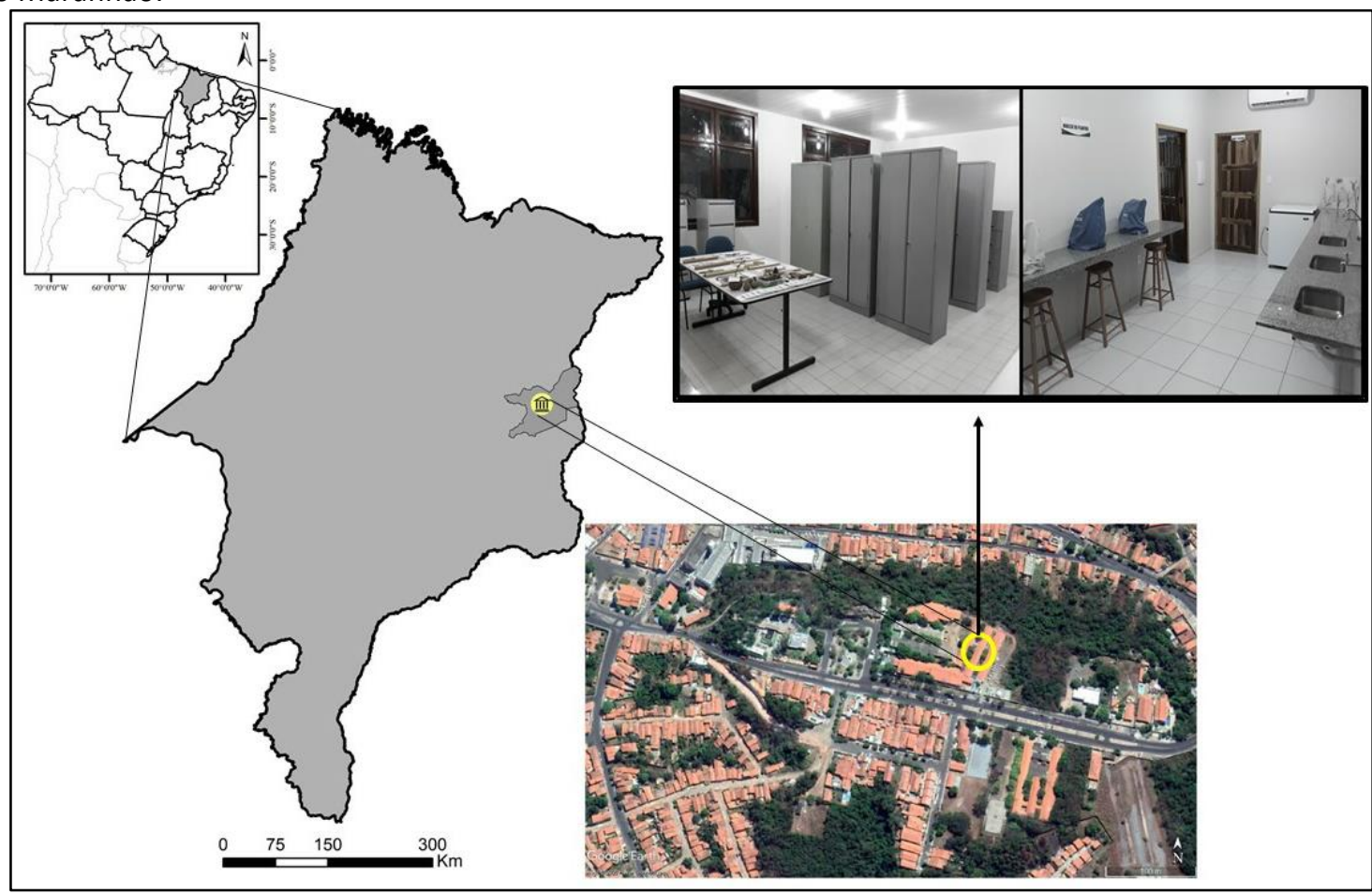


Alex Medeiros Silva, Domingos Lucas dos Santos-Silva, Guilherme Sousa da Silva, Gustavo da Silva Gomes, Regigláucia Rodrigues Oliveira, Ronison Ferreira Oliveira, Gonçalo Mendes da Conceição

O Index Herbariorum/IH lista 3324 herbários ativos no mundo, contendo 392.353.689 espécimes, fornecendo seus endereços, especialistas vinculados, principais coleções sob sua guarda e outras informações, e entre eles estão 180 herbários brasileiros, entre os quais, cinco do Maranhão. $\mathrm{O}$ registro do HABIT é uma conquista importante para a UEMA, uma vez que o IH registra apenas herbários com coleção suficientemente grande (cinco mil exemplares no mínimo), acessível aos cientistas e ativamente gerenciada.

A informatização dos dados do herbário é incentivada pelo projeto INCT Herbário Virtual de Plantas e Fungos do Brasil com o apoio do CNPq, o projeto visa integrar as ações e visões de curadores de herbários, pesquisadores, taxonomistas, programadores de sistemas de informação, disponibilizando dados e informações dos acervos botânicos, de forma livre e aberta, nas redes de informação (INCT, 2020). Com a iniciativa do INCT, desde 2018 informações são constantemente adicionadas do Herbário HABIT/UEMA e enviadas ao Herbário virtual (INCT).

A maior parte da coleção informatizada é de briófitas e os registros de angiospermas, samambaias e licófitas, aos poucos estão sendo adicionados, o que vale ressaltar, que com a parceria foi possível perceber a melhora da visibilidade dos dados e com a constante informatização a realização de mais coletas, contribuindo no reconhecimento da diversidade existente. É de grande relevância para o Maranhão a disponibilização dos dados do herbário ao Herbário virtual
(INCT), tal como, forma de registrar, compartilhar os dados entre herbários e listar as espécies das diferentes áreas de estudo da região, sendo importante no preenchimento de lacunas de conhecimentos da distribuição de espécies de briófitas para o Maranhão, região Nordeste e Brasil.

Os espécimes vegetais acervados no HABIT são de coletas realizadas por alunos e professores de graduação e pósgraduação em aulas de campo e atividades de pesquisa, bem como de doações de outros herbários de instituições nacionais e internacionais (QUEIRÓS et al. 2014; CONCEIÇÃO, 2015). Dentre os principais coletores do HABIT, tem-se G.M.Conceição, R.R.Oliveira, D.L.Santos-Silva, G.S.Silva e conta com a contribuição de especialistas de diferentes grupos taxonômicos e das áreas da biologia e ecologia vegetal (NYBG, 2020; SBB, 2020; CRIA, 2020).

Esses, por sua vez, se destacam pelos trabalhos científicos publicados e pelo acervo de espécies com novas ocorrências para o estado do Maranhão (Figura 3) (OLIVEIRA et al. 2018; SANTOS-SILVA et al. 2019; NASCIMENTO et al. 2020). Suprir as lacunas de conhecimento de registros das espécies é uma das metas para o total conhecimento da biodiversidade (HORTAL et al. 2015). No estado do Maranhão poucos trabalhos de cunho florístico são realizados e publicados, o que confere uma lacuna no conhecimento de registro de espécies, apesar dos últimos anos várias novas ocorrências têm sido publicadas e com a manutenção do herbário HABIT, essas iniciativas são melhores estabelecidas enriquecendo os dados para flora do

Figura 3. Espécimes depositados no herbário HABIT que categorizam novos registros para a Flora Maranhense. A. Folíolo de Fissidens crispus Mont. B. Gametófito de F. crispus. C. Folíolo de Fissidens lindbergii A. Jaeger. D. Gametófito de F. lindbergii. E. Schizaea elegans (Vahl) Sw. F. Cyclodium meniscioides (Willd.) C. Presl. G. Prestonia bahiensis Müll.Arg. H. Sida ciliares L. I. Sida urens L.

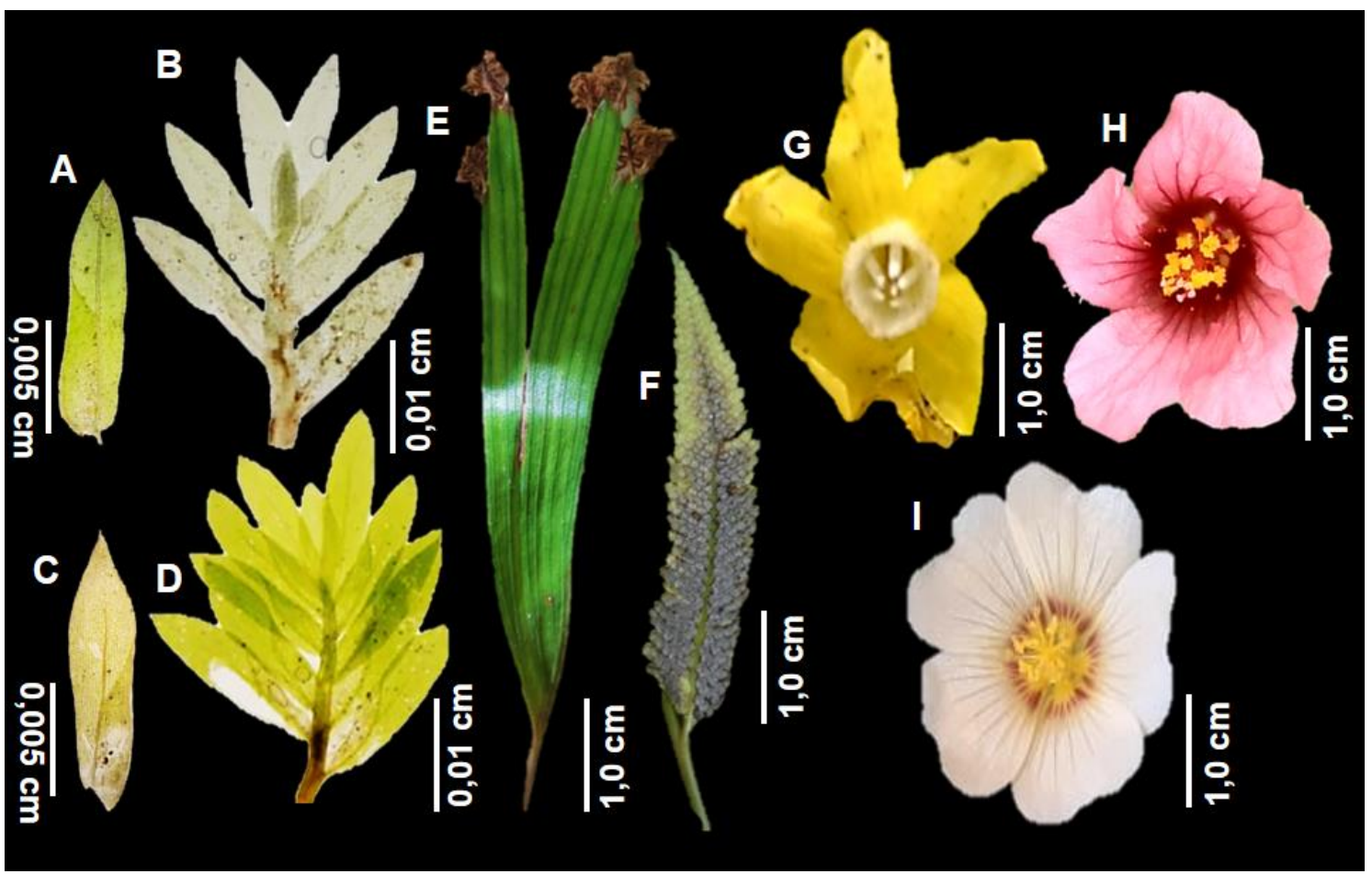


Maranhão e para os registros do herbário.

Com o reconhecimento de novos registros (Figura 3) para - Maranhão, amplia-se a distribuição geográfica desses táxons. Iniciativas como estas contribuem diretamente para o conhecimento da flora brasileira, consequentemente, cooperam para a elaboração de estratégias de conservação dos ambientes de ocorrência e de preservação das pequenas populações de espécies catalogadas até o momento (SANTOSSILVA et al. 2018)

\section{A Aplicabilidade do HABIT na Formação de Recursos Humanos e Científicos}

O HABIT tem contribuído na formação e desenvolvimento de jovens botânicos, taxonomistas e professores. Dessa forma, a aplicabilidade dos herbários vai muito além dos objetivos de criação, pois ao longo do tempo surgem novos usos para as coleções acervadas em herbários, na qual, são direcionadas pelos avanços científicos e necessidades da sociedade (FUNK, 2003; MASON HEBERLING; ISAAC, 2017; BESNARD et al. 2018).

O acervo botânico do HABIT é objetivo de estudo de muitas pesquisas científicas e de aperfeiçoamento profissional, como de trabalhos de conclusão de curso, projetos de iniciação científica, dissertações e de estágios. $O$ primeiro artigo disponível online sobre a coleção do HABIT, foi publicado em 2014, que se objetivou em elaborar um checklist das espécies de angiospermas doadas para o acervo botânico (QUEIRÓS et al. 2014). Em seguida, no ano de 2015, um outro artigo apresentou relatos das atividades e importância do HABIT (CONCEIÇÃO, 2015). Já os artigos dos anos de 2017 a 2018, apresentam uma lista de espécies da flora local e regional acervadas no HABIT, das famílias Fabaceae, Cyperaceae, Poaceae, Euphorbiaceae e Rubiaceae (DINIZ et al. 2017; MARTINS et al. 2017; NASCIMENTO et al. 2017; NASCIMENTO et al. 2018).

O HABIT além dos trabalhos científicos realizados internamente, também promove eventos de divulgação científica e interação com o meio acadêmico e comunidade em geral, contribuindo no fortalecimento da conservação e sensibilização ambiental. Como por exemplo, o BioBlitz, evento anual, realizado pela UEMA com o intuito de integrar professores, alunos e pesquisadores da instituição, em atividades interativas de campo com alunos de outras instituições de ensino básico e superior, onde a equipe do HABIT, quando convidada a participar do evento, demonstra

Figura 4. Atividades de extensão realizadas pela equipe do Herbário Professor Aluízio Bittencourt/HABIT. A) Trilha ecológica para visualização de espécies vegetais; B) Alunos em atividades de campo; C) Exposição e explicação de grupos vegetais acervado no HABIT; D) Visualização de estruturas botânicas na lupa.

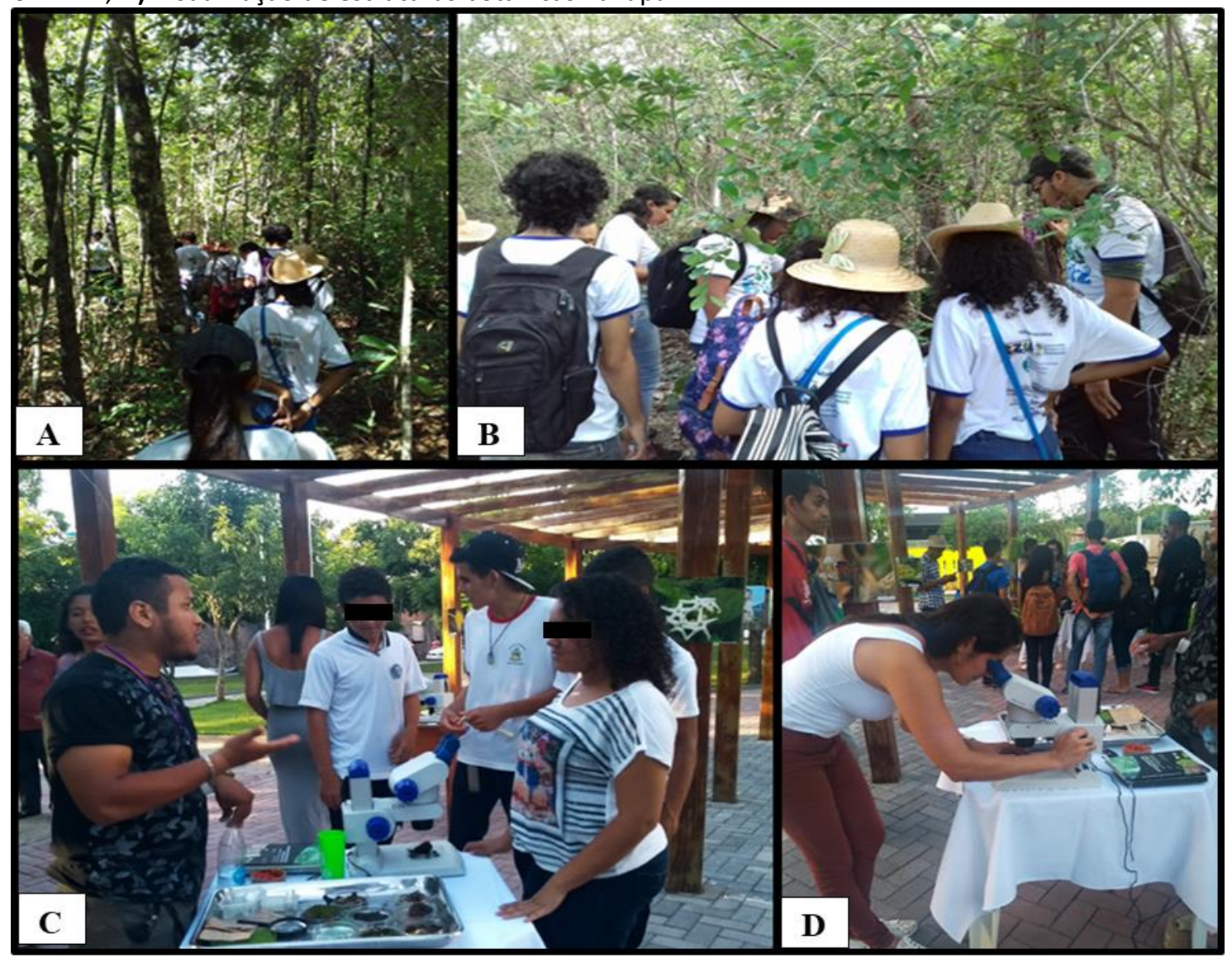


Alex Medeiros Silva, Domingos Lucas dos Santos-Silva, Guilherme Sousa da Silva, Gustavo da Silva Gomes, Regigláucia Rodrigues Oliveira, Ronison Ferreira Oliveira, Gonçalo Mendes da Conceição

Figura 5. Contribuições do Herbário Professor Aluízio Bittencourt/HABIT, para a conservação do patrimônio natural da biodiversidade do Maranhão.

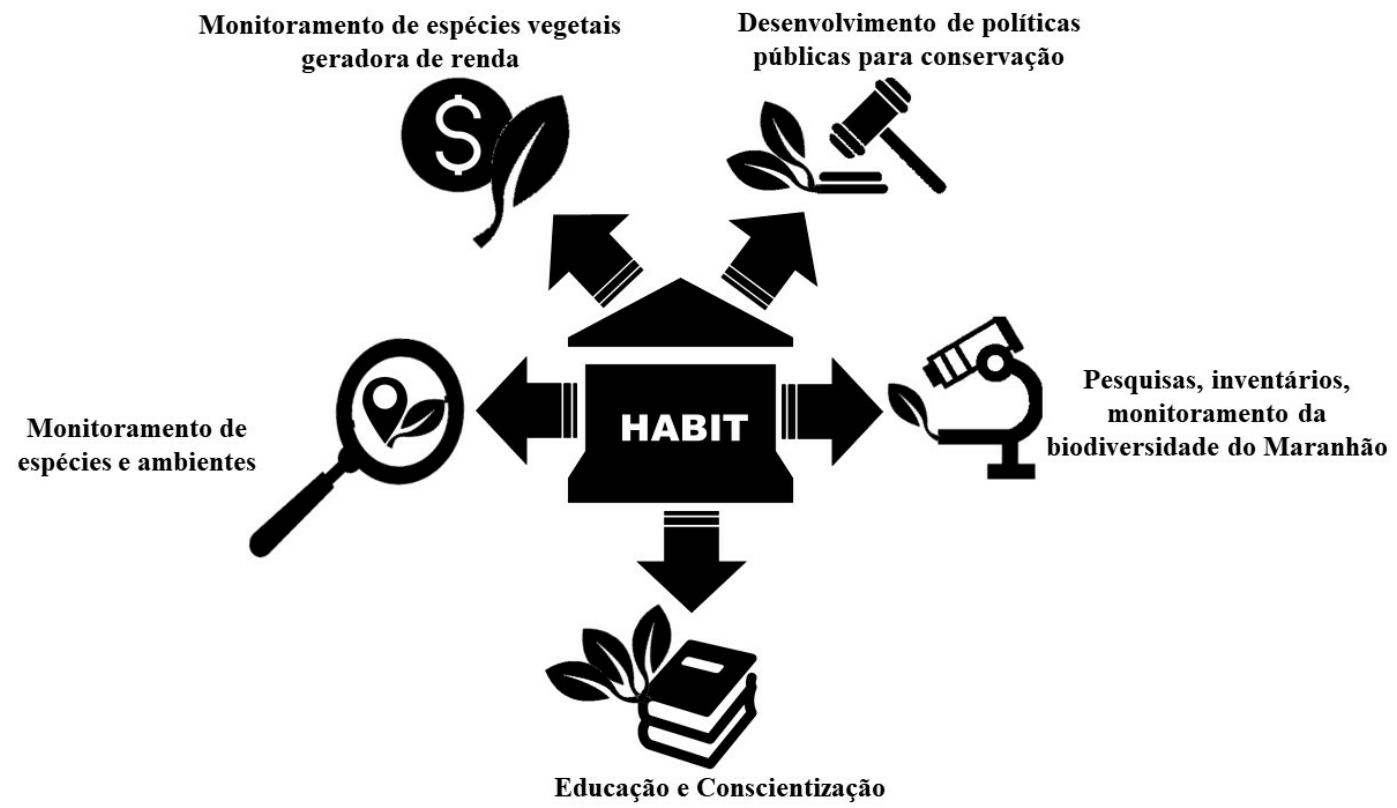

várias formas interativas com pesquisas de campo para o público-alvo, incentivando o interesse para área de botânica. São realizadas oficinas de botânicas, com procedimentos de coleta, herborização, montagem de exsicata e identificação de material botânico (Figura $4 \mathrm{~A}$ e B).

Outro evento realizado pelo HABIT destinado para alunos de escolas públicas e privadas, meio acadêmico e comunidade em geral, tem-se o Dia Mundial de Fascínio das Plantas (Fascination of Plants Day), com a intenção de mostrar a importância e beleza das plantas por meio de exposições de trabalhos científicos realizados pela equipe do LABIVE/HABIT, fotografias de espécies botânicas e ambientes de ocorrência, venda de plantas ornamentais, doação de amostras de livros e observação em microscópios e lupa de tecidos vegetais (Figura 4C e D).

Assim, as atividades realizadas pelo HABIT contribuem de fora direta e indireta para a conservação da biodiversidade local e regional do Maranhão. Onde o acervo do HABIT, que disponibiliza informações temporais (datas de coletas), espaciais (locais de coleta) e potencial de uso (dados econômicos) das espécies, podem auxiliar estudos ecológicos, de monitoramento e sobre os efeitos das mudanças climáticas na distribuição das espécies. Ainda possibilita trabalhar à educação ambiental, servindo como estratégia de sensibilização e conscientização da biodiversidade, devido ao HABIT oferecer oportunidades a comunidade geral, a conhecer e vivenciar a diversidade botânica local e regional, bem como, auxiliar na tomada de decisão política para conservar espécies e criar áreas de conservação (Figura 5).

\section{CONSIDERAÇÕES FINAIS}

O HABIT desempenha um papel fundamental para o conhecimento da flora local, regional, nacional e internacional para a todos os públicos, mediante a viabilidade de acesso, com a disponibilidade das informações online (Rede de Herbários). Além disso, as informações das amostras acervadas no HABIT, são fontes valiosas para pesquisadores, formuladores de políticas conservacionistas e para compreender a história natural dos estudos florísticos do Maranhão.

Mediante aos poucos estudos baseados na coleção do HABIT, reforça-se a necessidade de realização de trabalhos que se baseiam em listas florísticas das coleções inseridas no herbário, onde podem funcionar como documentos para desenvolver a biologia da conservação, em função das mudanças climáticas. Também são necessários o aperfeiçoamento de profissionais para atuarem em manutenção de coleções botânicas, frente aos avanços tecnológicos e científicos, para que os herbários possam continuar contribuindo com os objetivos usuais e potenciais. Consequentemente, é indispensável a aplicação de recursos financeiros em coleções botânicas para a catalogação e continuidade das atividades que estruturam, moldam e documentam o conhecimento da biodiversidade global.

\section{AGRADECIMENTOS}

Os autores agradecem ao INCT/CNPq a FAPEMA e a UEMA pela concessão de recursos ao HABIT e pela 
infraestrutura disponibilizada para coletas, herborização e identificação de plantas para o estado do Maranhão.

\section{REFERÊNCIAS}

ALMEIDA JR, E. B. de. Herbário do Maranhão, Maranhão (MAR). Herbários do Brasil - 66을 Congresso Nacional de Botânica. Unisanta Bioscience, v. 4, n. 6, p. 129-132, 2015.

ALVES, J. J. A.; ARAÚJO, M. A. DE; NASCIMENTO, S. S. do. Degradação da caatinga: uma investigação ecogeográfica. Caatinga, v. 22, n. 3, p. 126-135, 2009.

BESNARD, G.; GAUDEUL, M.; LAVERGNE, S.; MULLER, S.; ROUHAN, G. SUKHORUKOV, A. P.; VANDERPOORTEN, A.; JABBOUR, F. Herbarium-based science in the twenty-first century. Botany Letters, v. 165, n. 3-4, p. 323-327, 2018

CASTILLO-FIGUEROA, D. Beyond specimens: linking biological collections, functional ecology and biodiversity conservation. Revista peruana de biologia, v. 25, n. 3, p. $343-348,2018$.

CELENTANO, D.; MIRANDA, M. V. C.; MENDONÇA, E. N.; ROUSSEAU, G. X.; MUNIZ, F. H.; LOCH, V. do C.; VARGA, I. V. D.; FREITAS, L.; ARAÚJO, P.; NARVAES, I. da S.; ADAMI, M.; GOMES, A. R.; RODRIGUES, J. C.; KAHWAGE, C.; PINHEIRO, M.; MARTINS, M. B. Desmatamento, degradação e violência no "Mosaico Gurupi" - A região mais ameaçada da Amazônia. Estudos Avançados, v. 32, n. 92, 2018.

CONCEIÇÃO, G. M. da. Herbário Prof. Aluízio Bittencourt, Maranhão (HABIT). Herbários do Brasil - 66을 Congresso Nacional de Botânica. Unisanta Bioscience, v. 4, n. 6, p. 125-128, 2015.

COOK J., S.V. Edwards, E.A. Lacey.; Guralnick, R.P.; Soltis, P.S.; Soltis, D.E.; Welch, C.K.; Bell, K.C.; Galbreath, K.E.; Himes, C.; Allen, J.M.; Tracy A. Heath, T.A.; Carnaval, A.C.; Cooper, K.L.; Liu, M.; Hanken, J.; Ickert-Bond,S. Natural history collections as emerging resources for innovative education. BioScience. v. 64.p. 725-734, 2014.

CRIA - Centro de Referência em Informação Ambiental, 2020. Disponível em: http://splink.cria.org.br/manager/detail?resource=HABIT. Acesso em: 15 de abril 2020.

DINIZ, M. R.; SILVA, G. S. da; CONCEIÇÃO, G. M. da. Novas ocorrências para o Maranhão de espécies de Papilionoideae, depositadas no Herbário HABIT, do Centro de Estudos Superiores de Caxias, Maranhão. Biota Amazônia, Macapá, v. 7, n. 4, p. 57-59, 2017.

FUNK, V. 100 Uses for an Herbarium (Well at Least 72). American Society of Plant Taxonomists Newsletter, v. 17, n. 2, p. 17-19. 2003.

GREVE, M.; LYKKE, A.M.; FAGG, C.W.; GEREAU, R.E.; LEWIS,
G.P.; MARCHANT, R.; MARSHALLG, A.R.; NDAYISHIMIYE, J.; BOGAERT, J.; SVENNINGA, J. C. Realising the potential of herbarium records for conservation biology. South African Journal of Botany, v. 105, p. 317-323, 2016.

HORTAL, J.; BELLO, F.; DINIZ-FILHO, J. A. F.; LEWINSOHN, T. M.; LOBO, J. M.; LADLE, R. J. Seven Shortfalls that Beset LargeScale Knowledge of Biodiversity. The Annual Review of Ecology, Evolution, and Systematics, v. 46, p. 523-49, 2015.

INCT-Herbário Virtual da Flora e dos Fungos, 2020. Disponível em: http://inct.florabrasil.net/ Acesso em: 15 de abril 2020.

JOYE, D. A.; CASTELLA, E.; LACHAVANNE, E. J. B. Ocorrência de Characeae na Suíça nos últimos dois séculos (1800-2000). Botânica aquática, v. 72, n. 3, p. 369 - 385. 2002.

LE BRAS, G.; PIGNAL, M.; JEANSON, M.L.; MULLER, S.; AUPIC, C.; CARRÉ, B.; FLAMENT, G....HAEVERMANS, T. The French Muséum national d'histoire naturelle vascular plant herbarium collection dataset. Scientific Data, v. 4, e170016, 2017.

MARTINS, P. R. P.; SILVA, G. S. da; CONCEIÇÃO, G. M. da. Cyperaceae Juss. e Poaceae Barn. do Herbário Professor Aluízio Bittencourt da Universidade Estadual do Maranhão, Caxias, Brasil. Enciclopédia Biosfera, v. 14, n. 25, p. 1287, 2017.

MASON HEBERLING, J.; ISAAC, B. L. Herbarium Specimens as Exaptations: New Uses for Old Collections. American Journal of Botany, v. 104, n. 7, p. 963-965. 2017.

MUNIZ, F. H. A vegetação da região de transição entre a Amazônia e o Nordeste: diversidade e estrutura. In: Emanoel Gomes de Moura. (Org.). Agroambientes de transição entre o Trópico Úmido e o Semiárido do Brasil: atributos, alterações e uso na produção familiar. 2 eds. São Luís: Programa de Pós-graduação em Agroecologia/UEMA, 53-69. 2006.

NASCIMENTO, M. R.; SILVA, G. S. da; CONCEIÇÃO, G. M. da. Euphorbiaceae: Checklist de uma Coleção Botânica, Maranhão, Brasil. Enciclopédia Biosfera, v. 14, n. 25; p. 1353, 2017.

NASCIMENTO, J. de M.; VIANA, D. R.; SILVA, A. M.; SILVASANTOS, D. L.; OLIVEIRA, R. R. de; CONCEIÇÃO, G. M. de. Rubiaceae Juss. do Herbário Professor Aluízio Bittencourt, Maranhão, Brasil. Biota Amazônica, v. 8, n. 2, p. 24-31, 2018.

NASCIMENTO, J. de M.; GOMES, G. da S.; SILVA, G. S. da; SANTOS-SILVA, D. L. dos; ARAÚJO, M. de F. V.; CONCEIÇÃO G. M. Ampliando a Ocorrência de Malvaceae para o Maranhão, Brasil. Research, Society and Development, v. 9, n. 4, 2020.

NYBG - New York Botanical Garden, 2020. Disponível em: http://sweetgum.nybg.org/science/ih/. Acesso em: 15 de 
Alex Medeiros Silva, Domingos Lucas dos Santos-Silva, Guilherme Sousa da Silva, Gustavo da Silva Gomes, Regigláucia Rodrigues Oliveira, Ronison Ferreira Oliveira, Gonçalo Mendes da Conceição

Abril de 2020.

OLÍMPIO, A. P. M.; VENTURA, M. C. da S.; MASCARENHAS, M. de J. O.; NASCIMENTO, D. C. do; ANDRADE, F. A. G. de; FRAGA, E. da C.; BARROS, M. C. Bat fauna of the Cerrado savanna of eastern Maranhão, Brazil, with new species occurrences. Biota Neotropica, v. 16, n. 3, 2016.

OLIVEIRA, R. R. de; MEDEIROS, D. L.; OLIVEIRA, H. C. de; CONCEIÇÃO, G. M. da. Briófitas de área sob o domínio fitogeográfico do Cerrado e novas ocorrências para o Maranhão e região Nordeste do Brasil. Iheringia, Série Botânica, v. 73, n. 2, p. 191-195, 2018.

QUEIRÓS, S. G.; CONCEIÇÃO, G. M. da; NASCIMENTO, J. de M. Checklist das espécies de angiospermas doadas sob acervo, na Coleção Botânica do Laboratório de Biologia Vegetal, da Universidade Estadual do Maranhão. Agrarian Academy, v.1, n. 1, p. 285, 2014.

SANTOS-SILVA, D. L. dos; SILVA, G. S. da; OLIVEIRA, R. R. de; CONCEIÇÃO G. M. da. Nova Ocorrência de Lycopodiaceae (Lycophyta) para o Estado do Maranhão: Pseudolycopodiella carnosa (Silveira) Holub. Biota Amazônia, v. 8, n. 2, p. 58-59, 2018.

SANTOS-SILVA, D. L. dos; GOMES, G. da S.; SILVA, G. S. da; OLIVEIRA, R. F.; MARTINS, P. R. P.; SOUSA, D. H. S. de; ARAÚJO, M. de F. V.; CONCEIÇÃO, G. M. da. New occurrences of Schizaeaceae for the Maranhão and Brazilian Cerrado. International Journal of Development Research, v. 09, p. 26857-26862, 2019.

SEGOVIA-SALCEDO C.; CARRASCO, L.; ACOSTA-BUENAÑO, N. Las colecciones biológicas: Los tesoros escondidos de un país mega diverso. Revista ecuatoriana de medicina y ciencias biológicas. v.36. p. 83-88, 2015.

SBB - Sociedade Botânica do Brasil. Rede Brasileira de Herbários. Catalogo da Rede Brasileira de Herbários. 2020. Disponível em: <https://www.botanica.org.br/catalogoda-rede-brasileira-de-herbarios/>. Acesso em: 10 de Abril de 2020.

SILVA, E. O.; SOUSA, F. W. da S.; GUARÇONI, E. A. E.; FERREIRA, A. W. C.; JUNIOR, A. M.; MAGALHÃES, T. A. Implantação do Herbário do Curso de Ciências Naturais de Codó Prof. Deusiano Bandeira de Almeida (HENAC) - Relato de Caso. Enciclopédia Biosfera, v. 16, n. 29, p. 1881, 2019.

SIMMONS J.E.; MUÑOZ, Y. Cuidado, Manejo y Conservación de las Colecciones biológicas. Universidad Nacional de Colombia. 2005, 288pp. 